\title{
Measurement of Transverse Lambda Polarization in Quasi-Real Photoproduction at HERMES
}

\author{
Yu. Naryshkin \\ Petersburg Nuclear Physics Institute, \\ St. Petersburg, Gatchina, 188350 Russia
}

\begin{abstract}
Transverse $\Lambda$ and $\bar{\Lambda}$ polarization produced inclusively in quasi-real photon-nucleon scattering have been studied by the HERMES experiment using a $27.57 \mathrm{GeV}$ positron beam incident on hydrogen and deuterium gas targets. The average transverse polarizations were found to be $P_{n}^{\Lambda}=0.078 \pm 0.006$ (stat) \pm 0.012 (syst) and $P_{n}^{\bar{\Lambda}}=-0.025 \pm$ 0.015 (stat) \pm 0.018 (syst) for $\Lambda$ and $\bar{\Lambda}$ respectively. The dependences of $P_{n}^{\Lambda}$ on the transverse and longitudinal momenta of the $\Lambda$ hyperon were also studied.
\end{abstract}

\section{Introduction}

The polarization of $\Lambda$ particles has been observed and investigated in many high-energy scattering experiments, with a wide variety of hadron beams and kinematic settings $[2,3$, 4]. It is almost always found to be negative. A rather consistent kinematic behavior of the polarization has also been observed: its magnitude increases almost linearly with the transverse momentum $p_{\mathrm{T}}$ of the $\Lambda$ hyperon up to $p_{\mathrm{T}} \approx 4 \mathrm{GeV}$, where a plateau is reached. In photoproduction, the existing data are not conclusive because of lack of statistics. Here we report on the first statistically significant observation of non-zero transverse $\Lambda$ polarization in high energy (non-exclusive) photon scattering.

The $\Lambda$ hyperon is a uniquely useful particle in spin physics: the parity-violating nature of its weak decay $\Lambda \rightarrow p \pi^{-}$results in an angular distribution where the protons are preferentially emitted along the spin direction of their parent $\Lambda$. The angular distribution of the $\Lambda$ decay products may thus be used to measure its polarization, providing a rare opportunity to explore spin degrees of freedom in the fragmentation process. In the rest frame of the $\Lambda$ it has the form

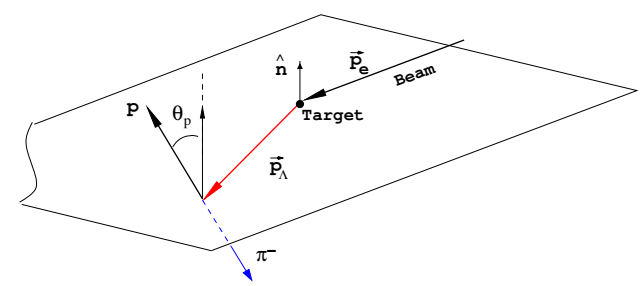

$$
\frac{d N}{d \Omega_{p}}=\frac{d N_{0}}{d \Omega_{p}}\left(1+\alpha P^{\Lambda} \cdot \cos \theta_{p}\right) .
$$

Here, $\theta_{p}$ is the angle between proton momentum and $\Lambda$ polarization direction in the

Figure 1: Schematic diagram of inclusive $\Lambda$ production and decay. The angle $\theta_{p}$ of the decay proton with respect to the normal $\hat{n}$ to the scattering plane is defined in the $\Lambda$ rest frame. $\Lambda$ rest frame (Fig.1), $P^{\Lambda}$ is the polarization of the $\Lambda$, and $\alpha=0.642 \pm 0.013$ is the analyzing power of the parity-violating weak decay. The symbols $d N / d \Omega_{p}$ and $d N_{0} / d \Omega_{p}$ denote the distributions for the decay of polarized and unpolarized $\Lambda$ samples respectively. Because of the parity-conserving nature of the strong interaction, any final-state hadron polarization in a reaction with unpolarized beam and target must point along a pseudo-vector direction. In the case of inclusive hyperon production, 
the only available direction of this type is the normal $\hat{n}$ to the scattering plane formed by the cross-product of the vectors along the laboratory-frame momenta of the positron beam $\left(\vec{p}_{e}\right)$ and the $\Lambda\left(\vec{p}_{\Lambda}\right)$ :

$$
\hat{n}=\frac{\vec{p}_{e} \times \vec{p}_{\Lambda}}{\left|\vec{p}_{e} \times \vec{p}_{\Lambda}\right|} .
$$

\section{The HERMES experiment and data analysis}

The $\Lambda$ photoproduction data were accumulated by the HERMES experiment at DESY. In this experiment, the $27.6 \mathrm{GeV}$ positron beam of the HERA $e-p$ collider passes through an open-ended tubular storage cell into which polarized or unpolarized target atoms in undiluted gaseous form are continuously injected. The HERMES detector is described in detail in Ref. [5].

This analysis combines the data collected at HERMES in the years 1996 2000. The sample includes data taken with both longitudinally polarized and unpolarized targets (the latter being of much higher density than the former), while the positron beam was always longitudinally polarized. As the target spin direction was reversed every 90 seconds, the average target polarization was negligibly small. The target species included hydrogen, deuterium, and a variety of heavier gases.

The $\Lambda$ hyperons were identified in the analysis through their $p \pi^{-}$decay channel. Events were selected by requiring the presence of at least two hadron candidates of opposite charge. The kinematics of the $\Lambda(\bar{\Lambda})$ decay products detected by the HERMES spectrometer is such that the proton (antiproton) momentum is always much higher than that of the pion. Two spatial vertices were reconstructed for each event. First the secondary (decay) vertex was determined
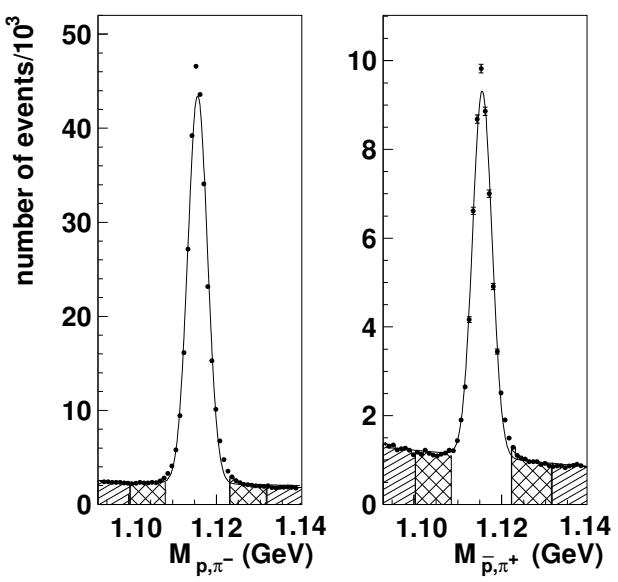

Figure 2: Invariant mass distributions for $\Lambda$ and $\bar{\Lambda}$ events. For the polarization analysis, $\Lambda$ and $\bar{\Lambda}$ events within a $\pm 3.3 \sigma$ invariant mass window around the mean value of the fitted peak were chosen, and a backgroundsubtraction procedure was applied.

from the intersection (i.e., point of closest

approach) of the proton (antiproton) and pion tracks. Then the intersection of the reconstructed hyperon track with the nominal beam axis was used to determined the primary (production) vertex. In both cases, the distance of closest approach was required to be less than $1.5 \mathrm{~cm}$. In addition the transverse distance between the decay vertex and the nominal beam axis was required to be larger than $1 \mathrm{~cm}$. If more than one positive or negative hadron was found in one event, all possible combinations of positive and negative hadrons were used.

All tracks were also required to satisfy a series of fiducial-volume cuts designed to avoid the inactive edges of the detector. Furthermore the two hadron tracks were required to be reconstructed in one spectrometer half to avoid effects caused by any possible misalignment of the two spectrometer halves relative to each other. For tracks fulfilling these requirements 
the invariant mass of the hadron pair was evaluated. The resulting spectra are shown in Fig.2.

The extraction of the $\Lambda$ polarization from the data was accomplished using a moment method which exploits the top/bottom symmetry of the detector and is described in Refs. [6, 7]. In order to study possible effects of detector misalignment and inefficiency detailed Monte-Carlo simulations were performed. The small contribution from the background under the $\Lambda$ invariant mass peak to the extracted polarizations was corrected for using a side-band subtraction method.

\section{Results}

Averaged over the experimental kinematics, the net $\Lambda$ polarization is found to be signficantly positive while the net $\bar{\Lambda}$ polarization is consistent with zero: $P_{\Lambda}=$ $0.078 \pm 0.006$ (stat) \pm 0.012 (syst). and $P_{\mathrm{n}}^{\bar{\Lambda}}=$ $-0.025 \pm 0.015$ (stat) \pm 0.018 (syst) [7].

In order to estimate the systematic uncertainty of the measurement an identical analysis was carried out for reconstructed $h^{+} h^{-}$hadron pairs, both with leading protons ( $\Lambda$-like case) and with leading antiprotons $(\bar{\Lambda}$-like case). Events within two mass windows above and below the $\Lambda(\bar{\Lambda})$ mass window $\left(1.093 \mathrm{GeV}<M_{h^{+} h^{-}}<\right.$ $1.108 \mathrm{GeV}$, and $1.124 \mathrm{GeV}<M_{h^{+} h^{-}}<$ $1.139 \mathrm{GeV}$ ) were selected with the hadrons' point of closest approach required to be inside the target region. False polarization values of $0.012 \pm 0.002$ and $0.018 \pm 0.002$ were found in the $\Lambda$-like and $\bar{\Lambda}$-like cases respectively. These values were used as estimates of the systematic error on the $\Lambda$ and $\bar{\Lambda}$ polarization. As an additional check
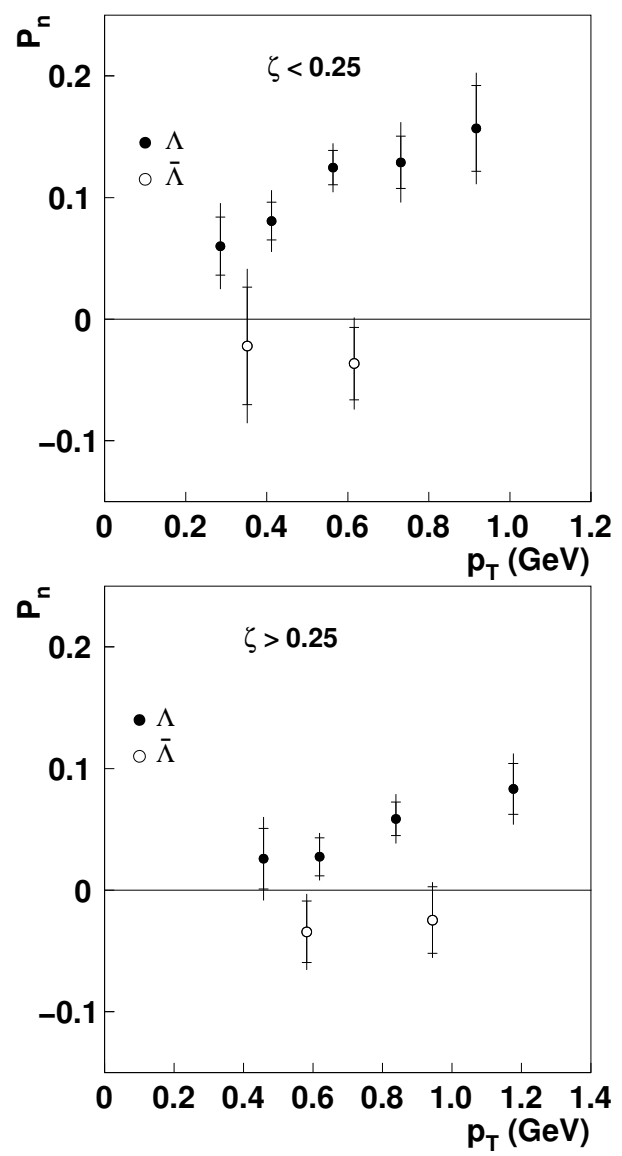

Figure 3: Transverse polarizations $P_{\Lambda}$ and $P_{\bar{\Lambda}}$ as a function of $p_{\mathrm{T}}$ for hyperons from the regions $\zeta<0.25$ (upper panel) and $\zeta>0.25$ (lower panel). The inner error bars represent the statistical uncertainties; the outer error bars represent the statistical and systematic uncertainties added in quadrature. on a possible false polarization, the decay $K_{s}^{0} \rightarrow \pi^{+} \pi^{-}$was studied. The false polarization of the $K_{s}^{0}$ sample was found to be $0.012 \pm 0.004$ and $0.002 \pm 0.004$ in the $\Lambda$-like and $\bar{\Lambda}$-like cases respectively.

As information on the virtual photon kinematics was not available in this inclusive measurement, the kinematic dependence of the polarization could only be studied as a function of variables derived from the $e N$ system. The selected variables were $p_{T}$ and 
$\zeta \equiv\left(E_{\Lambda}+p_{z \Lambda}\right) /\left(E_{e}+p_{e}\right)$, where $p_{T}$ is the transverse momentum with respect to the (lepton) beam, $E_{\Lambda}$ and $p_{z \Lambda}$ are the energy and $z$-component of the $\Lambda$ momentum (where the $z$-axis is defined as the lepton beam direction), and $E_{e}, p_{e}$ are the energy and momentum of the positron beam.

The variable $\zeta$ provides an approximate measure of whether a hyperon was produced in the forward or backward region in the center-of-mass frame of the $\gamma^{*} N$ reaction. The natural variable to use to separate these kinematic regimes would be $x_{\mathrm{F}}=p_{\|}^{\Lambda} / p_{\max }^{\Lambda}$, evaluated in the $\gamma^{*} N$ system, but this variable is not available in an inclusive measurement. Nevertheless, a simulation of the reaction using the PYTHIA program reveals a reasonable correlation between $\zeta$ and $x_{\mathrm{F}}$ variables. In particular, all events at $\zeta \geq 0.25$ are produced in the kinematic region $x_{\mathrm{F}}>0$, while for $\zeta<0.25$ there is a mixture of events originating from the kinematic regions $x_{\mathrm{F}}>0$ and $x_{\mathrm{F}}<0$. In Fig. 3, the transverse $\Lambda$ and $\bar{\Lambda}$ polarizations are shown versus $p_{\mathrm{T}}$ for the two intervals $\zeta<0.25$ and $\zeta>0.25$. In both regimes the $\Lambda$ polarization rises linearly with $p_{\mathrm{T}}$. The $\Lambda$ and $\bar{\Lambda}$ polarizations as functions of $\zeta$ are shown in Fig. 4 . The $\Lambda$ polarization appears to increase in the low- $\zeta$ region while the $\bar{\Lambda}$ polarization shows no visible dependence on

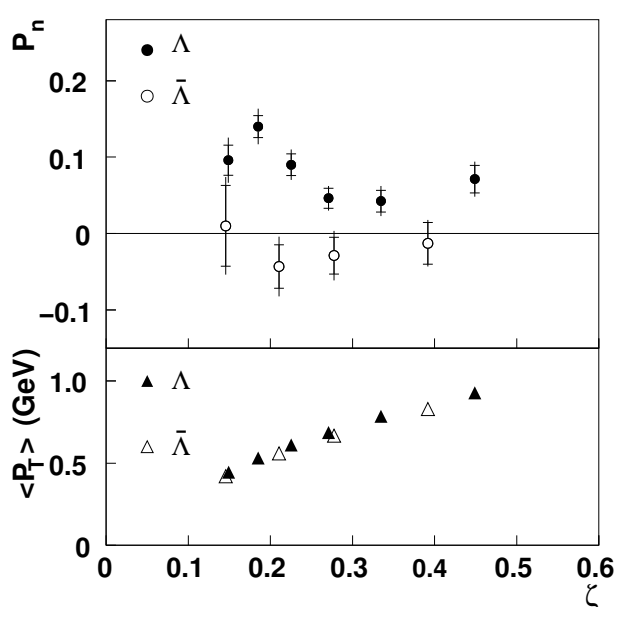

Figure 4: Transverse polarizations $P_{\Lambda}$ and $P_{\bar{\Lambda}}$ (upper panel) and mean $\left\langle p_{\mathrm{T}}\right\rangle$ (lower panel) as functions of $\zeta=\left(E_{\Lambda}+p_{z \Lambda}\right) /\left(E_{e}+p_{e}\right)$.

\section{Acknowledgments}

We gratefully acknowledge the DESY staff and the staffs of the collaborating institutions. This work was supported by the Russian Academy of Science and the Russian Federal Agency for Science and Innovations.

\section{References}

[1] Slides: http://indico.cern. ch/contributionDisplay.py? contribId=155\&sessionId=4\&conf $I d=9499$

[2] K. Heller, in "Proceedings of the $12^{\text {th }}$ International Symposium on High-Energy Spin Physics (SPIN 96)", edited by C.W. de Jager, T.J. Ketel, P.J. Mulders, J.E.J. Oberski, M. Oskam-Tamboezer (World Scientific, Singapore, 1997), p.23.

[3] J. Lach, Nucl. Phys. (Proc. Suppl.) 50216 (1996)

[4] WA89 Collaboration, M.I. Adamovich , Eur. Phys. J. C32 221 (2004)

[5] HERMES Collaboration, K. Ackerstaff et al., Nucl. Instrum. Methods A417 230 (1998).

[6] S. Belostotski DESY-HERMES-06-57.

Prepared for 58th Scottish Universities Summer School in Physics (SUSSP58): A NATO Advanced Study Institute and EU Hadron Physics 13 Summer Institute, St. Andrews, Scotland, 22-29 Aug 2004.

[7] A, Airapetian et al., arXiv:hep-ex/07043133 (2007). 